\title{
Breast augmentation complications following polyacrylamide hydrogel injection and fat grafting: Clinicopathologic report of 2 cases
}

Ghada Mohamed ${ }^{1,2^{*}}$, Mahitab El-Tohamy ${ }^{1,2}$, Mohamed Yousri ${ }^{1}$ and Mohammed Mohammed Gomaa ${ }^{3,4}$

*Correspondence: dr.ghada.elshafaee@gmail.com

CrossMark

$\leftarrow$ Click for updates

1Department of Pathology, National Cancer Institute, Cairo University, Cairo, Egypt.
2Department of Pathology, Baheya Foundation for Early Detection and Treatment of Breast Cancer, Giza, Egypt.
${ }^{3}$ Radiodiagnosis Department, National Cancer Institute, Cairo University, Cairo, Egypt.
${ }^{4}$ Radiodiagnosis Department, Baheya Foundation for Early Detection and Treatment of Breast Cancer, Giza, Egypt.

\begin{abstract}
Non-invasive procedure of breast augmentation is frequently demanded worldwide. Although several types of breast fillers are commercially available, none of them proved to be completely safe, with some reported long-term complications. The aim of this article is to report features of breast deformities in two patients, induced by two types of breast fillers; Polyacrylamide hydrogel (PAAG) and fat grafting. Despite PAAG has been prohibited in many countries, it is still being in use with undesirable complications. Both cases presented with painful breast masses and deformities. Although complications appeared long time after fat grafting, it developed more faster with PAAG injection. Sonomamographic examination followed by pathologic confirmation of the injected material were done. We consider that identification of breast augmentation complications is still a challenge to both pathologists and radiologists. However, proper history taken from the patient as well as the awareness of the microscopic features of different breast filler materials help in minimizing unnecessary further investigations and interventions.
\end{abstract}

Keywords: Breast augmentation, fat grafting, polyacrylamide hydrogel

\section{Background}

Breast augmentation is a frequently requested procedure in the plastic surgery in order to achieve the appropriate breast volume or to correct breast asymmetry that follows surgeries or occurs developmentally. Different procedures of breast enhancement were developed; invasive technique through implants insertion or flap surgeries, and non-invasive one using different breast fillers. The use of the latter became widely popular nowadays. Among different types of breast fillers; the use of Polyacrylamide hydrogel and fat grafting were commonly used [1,2].

Polyacrylamide hydrogel (PAAG) is a permanent filler composed of a hydrophilic gel of polyacrylamide that is extremely well tolerated and shows long duration of action [10]. However, the most common long-term complication of its use is the appearance of indurations, masses, deformity, pain,chronic infection, gel migration, cysts formations and adhesions [3].
Such complications can also affect radiologic visualization of the breast during screening and obscuring any developed mass, hence it delays cancer diagnosis. So far, five cases have developed breast cancer with history of PAAG injection [3]. However, there is no evidence that PAAG is a direct cause of breast malignancy.One of its most serious complication is pulmonary embolism which was reported as a consequence of gel migration [4]. PAAG products were prohibited in 2006 due to the growing numbers of the reported complications [5]. However few thousands of women were subjected to PAAG injection until 2019 as it was being sold illegally. For that reason, the distributer was then forced to cease the trade [6].

Fat grafting is another alternative, more safe and minimally invasive breast augmentation procedure. One of the largest systemic reviews indicated minimal complication following fat grafting including infection, cysts formation, fat necrosis and calcifications which develops several years after injection due

(c) 2021 Mohamed et al; licensee Herbert Publications Ltd. This is an Open Access article distributed under the terms of Creative Commons Attribution License (http://creativecommons.org/licenses/by/3.0). This permits unrestricted use, distribution, and reproduction in any medium, provided the original work is properly cited. 
to persistence of inflammation. There is no reported risk of cancer development [2]. Autologous fat grafting appears to be a safe procedure in breast augmentation, and it is widely used after mastectomies.

Herein we reported two different cases of breast enhancement using different breast fillers. There have been few published pathological reports worldwide describing the microscopic changes after breast enhancement, and none from Egypt. Being an unfamiliar subject to both pathologists and radiologists, it can mimic other breast diseases with unnecessary further investigations and overtreatment. We aimed in our report to highlight the clinical, radiologic and pathologic features of both circumstances.

\section{Case presentation}

This is a retrospective study of 2 cases who visited our hospital (Baheya Center) complaining of bilateral painful masses with history of two different types of breast fillers. One patient had PAAG injection into both breasts 3 years ago, while the other one had fat grafting 10 years ago. Both patients were subjected to bilateral Sono-mammography \& pathologic sampling of the detected lesions.

\section{CASE 1: Breast enhancement using Polyacrylamide hydrogel injections (PAAG)}

A 43-year-old woman with a family history of breast cancer (her grandmother) presented to our hospital on March 2021 complaining of bilateral breast deformity with painful masses of 2 years duration. She reported bilateral breast filler with PAAG hydrogel of 3 years duration. Clinical examination revealed bilateral breast tender masses. Mammography and ultrasonographic studies showed bilateral nearly symmetrical multiple isodense well circumscribed tubular-like structures showing internal densities content within. The largest mass appeared at the right breast directed towards 8 o'clock measuring about $5.5 \times 1 \mathrm{~cm}$ (BIRADS 3) (Figure 1), while the largest swelling at Lt. side is $5.5 \times 1.5 \mathrm{~cm}$.

Attempt at Lt. breast aspiration revealed thick and dried material. Then tissue biopsy was decided for proper diagnosis. Microscopic examination of the biopsy taken from the Rt. side showed breast tissue fragments with dilated cystic-like spaces. Cysts showed flattened lining \& filled with amorphous basophilic material with microvesicular appearance (Figures 2a and 2b). There were few foci of foreign-body giant cells reaction, set within a fibrotic stroma around cystic spaces (Figures $\mathbf{2 c}$ and $\mathbf{2 d}$ ). Microscopic findings confirmed no atypical or neoplastic cells. The apparent breast pathology was proved to be related to PAAG breast filler injection.

\section{CASE 2: Breast enhancement using Fat injection}

A 42 -year-old woman visited our hospital on June 2021 for bilateral breast masses and hard sensation of 5 months duration with breast contracture. The patient had history of bilateral breast fat grafting 10 years ago. Upon palpation, multiple

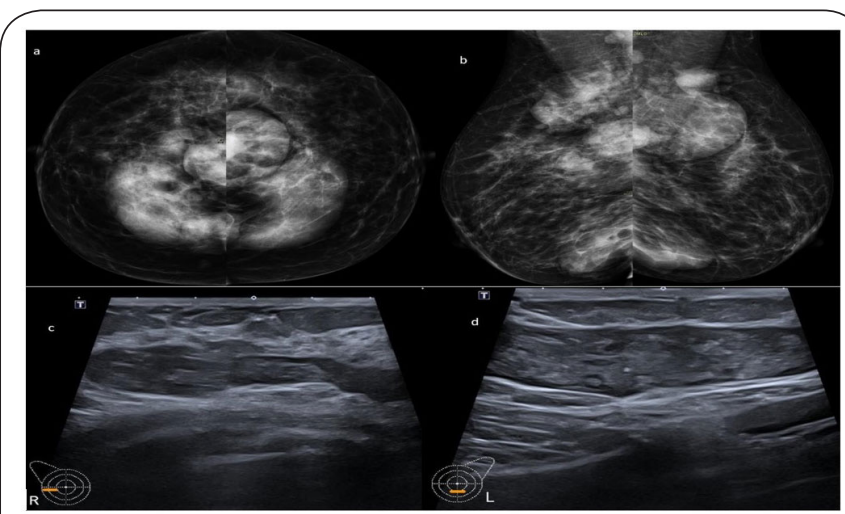

Figure 1. Radiologic findings: Post intramammary PAAG injection sonomammography.

Bilateral digital mammogram (a \& b): Bilateral nearly symmetrical multiple isodense well circumscribed lesions showing internal fat densities content within, suggestive of cystic masses. This was confirmed by

Complementary Ultrasound (c \& d): Bilateral tubular branching collections are seen of mixed echogenicity, the largest at the right breast is seen directed towards 8 oclock measuring about $5.5 \mathrm{xl} \mathrm{cm}$, and the largest at the left breast is seen directed towards 6 oclock measuring about $5.5 \times 1.5 \mathrm{~cm}$, (BIRADS 3).

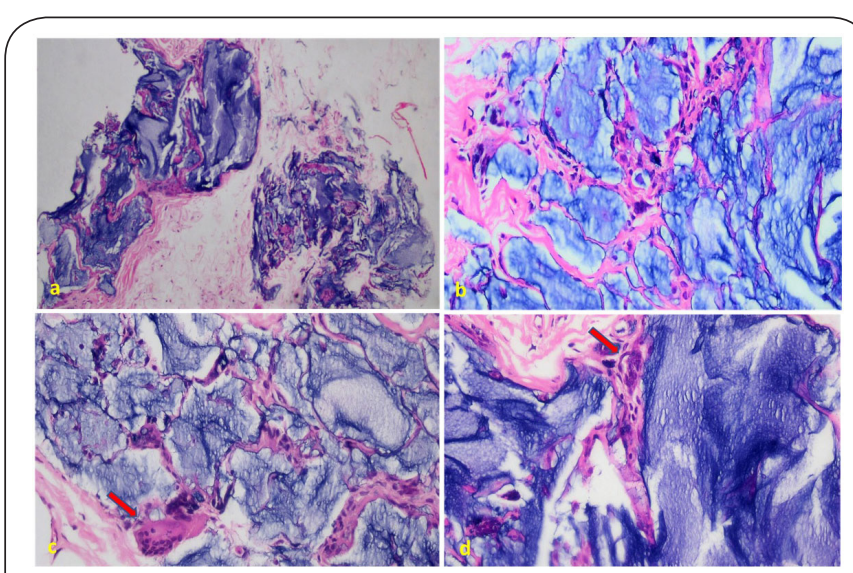

Figure 2: Hematoxylin and Eosin pathological image of PAAG injection for breast augmentation.

a $\& \mathbf{b}$ breast tissue fragments with dilated cystic-like spaces containing amorphous basophilic material with microvesicular appearance (PAAG material). a. HE. x40, b. HE. x100. c \& d Macrophages and multinucleated foreign body-type giant cells (arrows) are seen surrounding and directly related to the gel pools, c. HE. x100. d. HE. x200.

masses were identified in both breasts, accompanied by tenderness.

Bilateral sonomammography was performed \& showedheterogeneously dense mammary parenchyma with multiple bilateral circumscribed nearly symmetric hyperdense lesions corresponding by ultrasound to multiple scattered large complicated cystic lesions. Cysts occupied most of the breast parenchyma with small echogenic nodules, being the largest 
in the right side measured $8 \times 4 \mathrm{~cm}$, and that in the left side measured $7.7 \times 5.6 \mathrm{~cm}$ (BIRADS2) (Figure 3).

Right breast fine-needle aspiration was done. Cytologic examination revealed hypocellular smears with thick background secreted material. There were multiple scattered psammomatous calcifications with no atypical nor malignant cells (Figure 4). Trial of complete evacuation of the largest Cysts failed due to very thick content with obvious features of fat necrosis.

Both patients were then referred to the plastic surgery for further management.

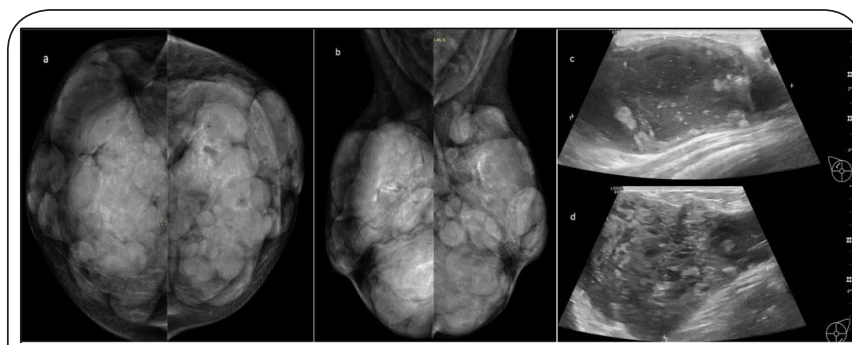

Figure 3. Radiologic findings: Post intramammary Fat injection sonomammography.

Bilateral Mammogram (a \& b) revealed: Heterogenously dense mammary parenchyma with multiple bilateral circumscribed nearly symmetric hyperdense lesions corresponding by ultrasound (c \& d) to multiple scattered large complicated cystic lesions with calcifications occupying most of the breast parenchyma showing predominantly hypoechgenicity with small echogenic nodules, the largest in the right side measured $8 \times 4 \mathrm{~cm}$, and in the left side measured $7.7 \times 5.6 \mathrm{~cm}$ (BIRADS 2).

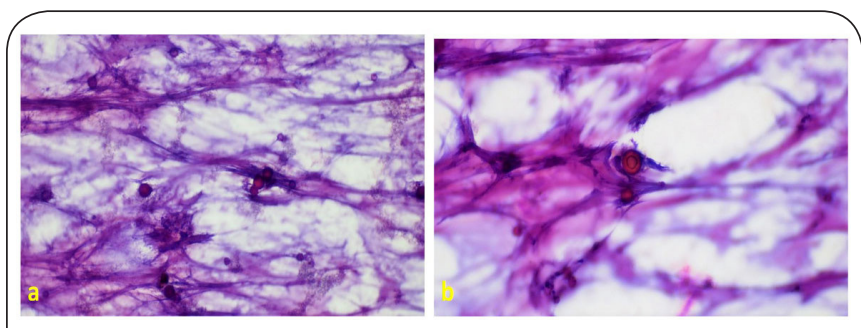

Figure 4: Cytologic features of the aspirated breast lesion with a history of fat injection for breast augmentation. a \& b: Multiple scattered psammomatous calcifications are seen in fine-needle aspiration of one of the complicated breast cysts. Papanicolaou stain, a. $\times 400$ \& b. x600.

\section{Discussion}

Breast augmentation using injectable fillers is one of the most popular physical enhancement surgeries, however with some reported complications. Different available commercial materials have been studied to overcome complications of the old injectable fillers, however with the appearance of some complications [7]. Polyacrylamide gel (PAAG) was one of the most commonly used fillers until it has been banned in China [5].
Our first case, had PAAG injection and the main reason for seeking medical advice was the development of breast masses and pain. It is known that PAAG injection should be done deeply into the subcutaneous fat, the muscle or into the supraperiosteal plane [8]. PAAG can be easily translocated into the subcautaneous tissue. The reported long-term complications, including deformities, lump appearance and skin irregularities, were mostly related to its leakage, superficial gel placement, uneven distribution or gel migration [3]. Treatment is usually through surgical intervention. However, it is usually difficult to do complete gel evacuation due to its extensive diffusion [9].

Reason for such cosmetic deformities is related to host reaction to the injected gel and formation of granulomatous foreign body reaction. Multinucleated giant cells appeared to surround the extracellular basophilic amorphous material (the gel material) [8].

Most of patients are not aware with the nature of the injected filler, others deny the history of breast augmentation. Histopathologic examination is the ideal way for confirmation of the exact type of breast filler. On microscopic examination, our case showed features similar to what described previously $[8,10,11]$. PAAG injection is characterized by formation of pale-to dark blue gel-containing pools of various sizes and shapes. It stained positive with Alcian blue stain and is not birefringent under the polarizing microscopy. Macrophages and variable amount of multinucleated foreign body giant cells are seen closely surrounding the gel pool [8]. A pseudocapsule can be formed and surrounds the filler [11].

Hyaluronic acid gel has a similar microscopic picture, however there is no multivacuolated appearance of the basophilic material, with less dense inflammatory granulomas than those appeared secondary to PAAG [8]. Other injectable filler shows different pictures. For example, injectable collagen which shows paucicellular eosinophilic material with rare granulomatous reaction. Poly-L-Lactic acid (PLLA) is characterized histologically by an eosinophilic fusiform or spiky material that polarizes readily [12].

Mucin-rich breast lesions, with a basophilic background similar to PAAG gel,also come in the differential diagnosis. This includes mucinous carcinoma, cystic hypersecretory breast lesions, and mucocele-like lesions. Detailed described pathologic features of these disease can help in the differential diagnosis $[13,14]$.

Radiologic examination has an important role for diagnosis and treatment of breast complication after augmentation, especially when radiologist is aware with this procedure. While ultrasound is usually used for localization of the gel nodules and aspiration of the inflamed collection, MRI is the most sensitive technique for assessing the volume and extent of injectable PAAG [15].

Fat grafting is a minimally invasive and a safe procedure for breast augmentation with few reported complications and no risk for breast cancer development [15]. The most common 
complication arising from fat grafting is fat necrosis, which causes calcifications and oil cysts. Dead adipocytes become oil droplets, when it is in a large amount it is difficult to be absorbed. Oil cysts are formed 6-12 months after grafting [16]. Macrophages are then surround oil droplet with the formation of fibrous capsule that stops oil absorption. Calcifications of the wall progress over years due to persistence of the inflammation [17]. Symptoms appeared in relation to this process include lump formation, tenderness and contracture. Other reported serious complication from fat grafting is arterial embolization and stroke [18].

Two forms of calcifications appeared with fat grafting. Small fat necrosis leads to formation of sand-like calcifications over years, while large amount of fat necrosis forms oil cyst with fibrous capsule that is classified after long time forming an egg-shell like calcifications [16].

In our case, the complications appeared after 10 years of bilateral breast injection in the form of breast masses with hard breast sensation and bilateral breast contracture. The radiologic appearance of multiple cystic lesions together with the appearance of calcifications on cytologic examination denoted fat necrosis with formation of oil cystsand calcifications. Once oil cyst is formed, a progressive course of endless inflammation and calcification occur [17].

Most of radiologic changes following fat grafting are in the form of oil cysts, calcifications and fat necrosis, which are easily distinguishable from malignant changes [2]. Reason for such complication is mainly due to the injection technique and amount of fat grafting. Surgeon should be cautious during fat grafting to avoid unexcepted breast damage. Treatment will be usually through surgical intervention or alternatively cutting the oil cyst with squeezing its content leading to its phagocytosis [16].

In conclusion, our report showed that despite PAAG has been prohibited in many countries, it is still being in use with undesirable complications. We strongly recommend the need for increase public awareness about complications of breast augmentation with emphasis on the prohibited materials. Although fat grafting is still considered the safest enhancement technique with less complications, we and others still reporting the appearance of long-term complications. Hence, there is a need for more safe breast augmentation techniques and materials. Histopathologic examination remains to be the gold standard way in identifying type of breast filler. Most of pathologists and radiologists are unfamiliar with filler materials and complications, thus we hope that our report illuminated this issue with the possible differential diagnosis.

\section{List of abbreviations}

PAAG: Polyacrylamide hydrogel

PLLA: Poly-L-Lactic acid

MRI: Magnetic resonance imaging

Competing interests

The authors declare that they have no competing interests.

\section{Authors' contributions}

\begin{tabular}{|l|c|c|c|c|}
\hline Authors' contributions & GM & ME & MY & MMG \\
\hline Research concept and design & $\sqrt{ }$ & -- & -- & -- \\
\hline Collection and/or assembly of data & $\sqrt{ }$ & -- & $\sqrt{ }$ & $\sqrt{ }$ \\
\hline Data analysis and interpretation & $\sqrt{ }$ & $\sqrt{ }$ & $\sqrt{ }$ & $\sqrt{ }$ \\
\hline Writing the article & $\sqrt{ }$ & $\sqrt{ }$ & -- & $\sqrt{ }$ \\
\hline Critical revision of the article & $\sqrt{ }$ & $\sqrt{ }$ & -- & -- \\
\hline Final approval of article & $\sqrt{ }$ & $\sqrt{ }$ & $\sqrt{ }$ & $\sqrt{ }$ \\
\hline Statistical analysis & -- & -- & -- & -- \\
\hline
\end{tabular}

Acknowledgments

We would like to acknowledge all biotechnologists in the Pathology Department at Baheya Center for their help on staining and preparing the samples.

Publication history

Editor: Khush Mittal, New York University School of Medicine, USA. Received: 12-Oct-2021 Final Revised: 15-Nov-2021

Accepted: 19-Dec-2021 Published: 31-Dec-2021

\section{References}

1. Du F, Wang $X$, Wang $Z$, Huang $W$. The long-term results of polyacrylamide hydrogel for augmentation mammoplasty. Journal of Plastic, Reconstructive \& Aesthetic Surgery. 2019,1;72(12):2064-94.

2. Orholt M, Larsen A, Hemmingsen MN, Mirian C, Zocchi ML, VesterGlowinski PV, Herly M. Complications after breast augmentation with fat grafting: a systematic review. Plastic and reconstructive surgery. 2020, 1;145(3):530e-7e.

3. Unukovych D, Khrapach V, Wickman M, Liljegren A, Mishalov V, Patlazhan $\mathrm{G}$, et al. Polyacrylamide gel injections for breast augmentation: management of complications in 106 patients, a multicenter study. World J Surg. 2012,36(4):695-701.

4. Duan Y, Zhang L, Li S, Yang Y, Xing J, Li W, Wang X, Zhou Y. Polyacrylamide hydrogel pulmonary embolism - a fatal consequence of an illegal cosmetic vaginal tightening procedure: a case report.Forensic science international. 2014,1;238:e6-10.

5. China Food and Drug Administration [cited 2010 September 03]. Available from: http://samr.cfda.sda.gov.cn/WS01/CL1994/53328.html.

6. Statement of the Director of the Office for Registration of Medical Products, Medicinal Devices and Biocidal Products in Poland (2020) No. UR.D.WM_.DNB_.5.2020z27.01.2020r.pdf. https://www.urpl.gov. $\mathrm{pl} /$ sites/default/files/Decyzja\%20UR.D.WM_.DNB_.5.2020\%20z\%20 27.01.2020\%20r.pdf

7. Nomoto S, Hirakawa K, Ogawa R. Safety of Copolyamide Filler Injection for Breast Augmentation.Plastic and Reconstructive Surgery Global Open. 2021, 9(2).

8. Requena L, Requena C, Christensen L, Zimmermann US, Kutzner H, Cerroni L. Adverse reactions to injectable soft tissue fillers. Journal of the American Academy of Dermatology. 2011, 1;64(1):1-34.

9. Kim HJ, Lee SJ, Lee JH, Shin SH, Kim SH, Kim JH, Suh IS. Breast reconstruction after complications following breast augmentation with massive filler injections. Medicine. 2020, 14;99(33).

10. Wang Z, Li S, Wang L, Zhang S, Jiang Y, Chen J, Luo D. Polyacrylamide hydrogel injection for breast augmentation: another injectable failure. Medical science monitor: international medical journal of experimental and clinical research. 2012,18(6):CR399.

11. Yang Y, Li S, He J, Zhao X, Chen W, Dai X, Liu L. Clinicopathological analysis of $\mathbf{9 0}$ cases of polyacrylamide hydrogel injection for breast augmentation including $\mathbf{2}$ cases followed by breast cancer.Breast Care. 2020,15(1):38-44.

12. Rebecca Kleinerman MD, Gary Goldenberg MD, Emanuel PO. Histopathologic identification of dermal filler agents. Journal of Drugs in Dermatology. 2010,9(9).

13. Mohamed G, EIMahs A and Ahmed H. A rare case of invasive cystic 
Mohamed et al., Journal of Histology \& Histopathology 2021, http://www.hoajonline.com/journals/pdf/2055-091X-8-5.pdf

hypersecretory carcinoma of the breast post neoadjuvant therapy: Case report and literature review. J HistolHistopathol. 2021, 8:2.

14. Tan PH, Ellis I, Allison K, Brogi E, Fox SB, Lakhani S, Lazar AJ, Morris EA, Sahin A, Salgado R, Sapino A, Sasano H, Schnitt S, Sotiriou C, van Diest P, White VA, Lokuhetty D, Cree IA; WHO Classification of Tumours Editorial Board. The $\mathbf{2 0 1 9}$ World Health Organization classification of tumours of the breast.Histopathology. 2020,77(2):181-185.

15. Hedher NB, David J, Trop I, Drouin S, Peloquin L, Lalonde L. Imaging findings of breast augmentation with injected hydrophilic polyacrylamide gel: patient reports and literature review. Eur J Radiol. 2011,78(1):104-11

16. Yoshimura K, Coleman SR. Complications of Fat Grafting: How They Occur and How to Find, Avoid, and Treat Them.Clin Plast Surg. 2015,42(3):383-8.

17. Mineda $\mathrm{K}$, Kuno $\mathrm{S}$, Kato $\mathrm{H}$, et al. Chronic inflammation and progressive calcification as a result of fat necrosis: the worst outcome in fat grafting. PlastReconstr Surg 2014,133:1064-72.

18. Coleman SR. Avoidance of arterial occlusion from injection of soft tissue fillers.Aesthet Surg J 2002,22:555-7.

\section{Citation:}

Mohamed G, El-Tohamy M, Yousri M and Gomaa MM. Breast augmentation complications following polyacrylamide hydrogel injection and fat grafting: Clinicopathologic report of 2 cases. J Histol Histopathol. 2021; 8:5. http://dx.doi.org/10.7243/2055-091X-8-5 